\title{
DETERMINANT EXPRESSION OF SELBERG ZETA FUNCTIONS. II
}

\author{
SHIN-YA KOYAMA
}

\begin{abstract}
This paper is the $\operatorname{PSL}(2, \mathbf{C})$-version of Part I. We show that for $\operatorname{PSL}(2, \mathrm{C})$ and its subgroup $\operatorname{PSL}(2, O)$, the Selberg zeta function with its gamma factors is expressed as the determinant of the Laplacians, where $O$ is the integer ring of an imaginary quadratic field. All the gamma factors are calculated explicitly. We also give an explicit computation to the contribution of the continuous spectrum to the determinant of the Laplacian.
\end{abstract}

\section{INTRODUCTION}

In Part I [6], we expressed the Selberg zeta function as the determinant of the Laplacian for $G=\operatorname{PSL}(2, \mathbf{R})$ and its congruence subgroup $\Gamma$. In this paper, we will show that the same type of expression is valid for $G=\operatorname{PSL}(2, \mathbf{C})$ and $\Gamma=\operatorname{PSL}\left(2, O_{K}\right)$, where $O_{K}$ is the integer ring of an imaginary quadratic field $K$. The Selberg zeta function is defined by

$$
Z(s):=\prod_{T \in \mathbf{P}} \prod_{(k, l)}\left(1-a(T)^{-2 k} \overline{a(T)}^{-2 l} N(T)^{-s}\right),
$$

where $\mathbf{P}$ is a certain set of primitive hyperbolic conjugacy classes and $(k, l)$ runs through all the pairs of positive integers satisfying a certain congruence relation. The complex number $a(T)$ is the eigenvalue of $T$ with $|a(T)|>1$, and $N(T):=|a(T)|^{2}$. By supplying three factors to $Z(s)$, we have the complete Selberg zeta function

$$
\widehat{Z}(s):=Z_{I}(s) Z_{E}(s) Z_{P}(s) Z(s),
$$

where $I, E$, and $P$ correspond to the contribution of the identity, elliptic, and parabolic conjugacy classes, respectively. The main result of this paper is that $\widehat{Z}(s)$ has the determinant expression

$$
\widehat{Z}(s)=e^{c-c^{\prime} s(2-s)} \operatorname{det}(\Delta, s),
$$

where $\Delta$ is the Laplacian for the real three-dimensional hyperbolic space. The determinant of the Laplacian is composed of both discrete and continuous spectrum:

$$
\operatorname{det}(\Delta, s):=\operatorname{det}_{D}(\Delta-s(2-s)) \operatorname{det}_{C}(\Delta, s) .
$$

Received by the editors July 14, 1989 and, in revised form, November 29, 1989.

1980 Mathematics Subject Classification (1985 Revision). Primary 11F72. 
The discrete part $\operatorname{det}_{D}$ is defined via the spectral zeta function, while the continuous part $\operatorname{det}_{C}$ is computed from the corresponding terms of the Selberg trace formula. An explicit computation shows that $Z_{I}$ and $Z_{E}$ are entire, $Z_{P}$ is expressed by the gamma function, and $\operatorname{det}_{C}$ is the Dedekind zeta function with the gamma factor (the complete Dedekind zeta function) of the Hilbert class field of $K$. We can list all the zeros of $Z(s)$ via the eigenvalues of $\Delta$ and the zeros of the Dedekind zeta function. When $G=\operatorname{PSL}(2, \mathbf{R})$, zeros of $Z(s)$ deriving from $Z_{I}(s)^{-1} Z_{E}(s)^{-1}$ are called trivial zeros. In the present situation, $Z(s)$ has no trivial zeros. This reflects the fact that there is no discrete series among the representations of $\operatorname{SL}(2, C)$. In the last section, we give an account of the work of Efrat in [1], which suggests a general definition of the determinant of the Laplacians, and we put the results in the former sections in this paper into his general framework.

The author would like to express his profound gratitude to Professor N. Kurokawa, who introduced the author to the subject and gave many useful suggestions and encouragement to the author in the entire process.

\section{The Selberg trace formula}

Let $j$ be the number in the quaternion field which satisfies $j^{2}=-1, i j=$ $-j i$, and let $\mathbf{H}$ be the real three-dimensional hyperbolic space,

$$
\mathbf{H}:=\left\{v=z+y j \mid z=x_{1}+x_{2} i \in \mathbf{C}, y>0\right\},
$$

with the Riemannian metric

$$
d v^{2}=\frac{d x_{1}^{2}+d x_{2}^{2}+d y^{2}}{y^{2}} .
$$

It has a corresponding hyperbolic distance $d\left(v, v^{\prime}\right)$ given by

$$
\cosh d\left(v, v^{\prime}\right):=\frac{\left|z-z^{\prime}\right|^{2}+y^{2}+y^{\prime 2}}{2 y y^{\prime}},
$$

where $v=z+y j$ and $v^{\prime}=z^{\prime}+y^{\prime} j$. Moreover, the hyperbolic volume measure is given by

$$
\frac{d x_{1} d x_{2} d y}{y^{3}} .
$$

The group $\operatorname{SL}(2, \mathbf{C})$ acts on $\mathbf{H}$ transitively by

$$
\left(\begin{array}{ll}
a & b \\
c & d
\end{array}\right)(v):=(a v+b)(c v+d)^{-1}=\frac{(a z+b) \overline{(c z+d)}+a \bar{c} y^{2}+y j}{|c z+d|^{2}+|c|^{2} y^{2}} .
$$

The induced action of $G=\operatorname{PSL}(2, \mathbf{C})$ is faithful and the stabilizer of $j$ is the unitary group $\mathrm{SU}(2)$, which induces the analytic isomorphism

$$
g M \in G / M \rightarrow g(j) \in \mathbf{H},
$$

where $M:=\mathrm{SU}(2) /\left\{ \pm 1_{2}\right\}$. We introduce an imaginary quadratic field $K=$ $\mathbf{Q}(\sqrt{-D})$, where $D$ is a square free positive integer such that $D \neq 1,3$. Let $\Gamma$ be $\operatorname{PSL}\left(2, O_{K}\right)$, which is a discrete subgroup of $G$. The Laplacian for $\mathbf{H}$ is defined by

$$
\Delta:=-y^{2}\left(\frac{\partial^{2}}{\partial x_{1}^{2}}+\frac{\partial^{2}}{\partial x_{2}^{2}}+\frac{\partial^{2}}{\partial y^{2}}\right)+y \frac{\partial}{\partial y} .
$$


It has the selfadjoint extension on $L^{2}(\Gamma \backslash \mathbf{H})$. For the right regular representation $U$ of $G$ on $L^{2}(\Gamma \backslash G)$ and the function $f \in L^{1}(G)$, we define the operator $U(f)$ on $L^{2}(\Gamma \backslash G)$ by

$$
U(f):=\int_{G} f(y) U(y) d y .
$$

The operator $U(f)$ has both discrete and continuous spectrum. The Selberg trace formula expresses the sum of the discrete spectrum as

$$
\left.\operatorname{Tr}_{D}=\text { (sum over conjugacy classes }\right)-\operatorname{Tr}_{C} \text {, }
$$

where $\operatorname{Tr}_{C}$ corresponds to the removed trace of the continuous spectrum. If we denote the eigenvalues of $\Delta$ by $\left\{0=\lambda_{0}<\lambda_{1}<\lambda_{2}<\cdots\right\}$, then the eigenvalues of $U(f)$ depend only on $\left\{\lambda_{n}\right\}$. So we have

$$
\operatorname{Tr}_{D}=\sum_{n=0}^{\infty} h\left(\lambda_{n}\right)
$$

The function $h\left(\lambda_{n}\right)$ is called the Selberg transform of $f$. Next we give the classification of conjugacy classes. An element $\gamma \in \Gamma-\{1\}$ is called elliptic, hyperbolic, or parabolic if $|\operatorname{tr}(\gamma)|$ is smaller than, larger than, or equal to 2, respectively. The norm of a hyperbolic element $\gamma$ is defined by $N(\gamma)=|\alpha|^{2}$, if $\alpha \in \mathbf{C}$ is the eigenvalue of $\gamma$ such that $|\alpha|>1$. The centralizer $\Gamma_{\gamma}$ of a semisimple element $\gamma$ in $\Gamma$ is given by the following lemmas.

Lemma 2.1 (Elstrodt et al. [3, Theorem 2.1, p. 96]). Suppose that $R \in \Gamma$ is elliptic, and let $R_{0}$ be a primitive elliptic element associated with $R$. Then the centralizer $\Gamma_{R}$ of $R$ in $\Gamma$ contains hyperbolic elements. Let $T_{0} \in \Gamma_{R}$ be hyperbolic such that $N\left(T_{0}\right)$ is minimal in the set of norms of hyperbolic elements contained in $\Gamma_{R}$. Then one of the two following possibilities occurs:

(a) $\left\langle R_{0}\right\rangle$ contains all the elliptic elements of $\Gamma_{R}$. Then $\Gamma_{R}$ is abelian,

$$
\Gamma_{R}=\left\langle R_{0}\right\rangle \times\left\langle T_{0}\right\rangle,
$$

and $M(R):=\left\langle R_{0}\right\rangle$ is the unique maximal finite subgroup of $\Gamma_{R}$.

(b) $R$ is elliptic of order 2 , and there exists an elliptic element $S \in \Gamma_{R}$ also of order 2. Then for every such $S$

$$
S^{-1} R_{0} S=R_{0}^{-1}, \quad \text { and } \quad M(R):=\left\langle R_{0}\right\rangle \cup\left\langle R_{0}\right\rangle S
$$

is a maximal finite subgroup of $\Gamma_{R}$. All the maximal finite subgroups of $\Gamma_{R}$ are conjugate in $\operatorname{PSL}(2, \mathbf{C})$,

$$
\Gamma_{R}=\left\{T_{0}^{n} E \mid E \in M(R), n \in \mathbf{Z}\right\},
$$

and $\left\langle R_{0}\right\rangle \times\left\langle T_{0}\right\rangle$ is an abelian subgroup of index 2 in $\Gamma_{R}$.

Lemma 2.2 (Elstrodt et al. [3, p. 94]). Suppose that $T \in \Gamma$ is hyperbolic, and let $\left(\Gamma_{T}\right)_{\text {tor }}$ be the set of elements of finite order in $\Gamma_{T}$. Then $\left(\Gamma_{T}\right)_{\text {tor }}$ contains only the identity or it is the finite cyclic group generated by the hyperbolic rotation in $\Gamma$ with minimal rotation angle around the axis of $T$. Let $T_{0}$ be an element such that $N\left(T_{0}\right)$ is minimal among the set of norms of all hyperbolic elements of $\Gamma_{T} . T_{0}$ itself is not uniquely determined by $T$, but $N\left(T_{0}\right)$ is. Then

$$
\Gamma_{T}=\left(\Gamma_{T}\right)_{\mathrm{tor}} \times\left\langle T_{0}\right\rangle .
$$

In particular, $\Gamma_{T}$ is abelian. 
Remark. These lemmas are proved for $\Gamma$ cocompact, but valid for $\Gamma$ cofinite, because parabolic elements have no effect on the centralizer of a semisimple element.

Here we choose a maximal system $\mathbf{P}$ of primitive hyperbolic elements of $\Gamma$ such that no two of the elements

$$
T=T_{0}^{n+1} R_{0}^{m} \quad\left(T_{0} \in \mathbf{P}, M\left(T_{0}\right)=\left\langle R_{0}\right\rangle, 0 \leq m<\operatorname{ord} R_{0}, n \geq 0\right)
$$

are conjugate in $\Gamma$.

Lemma 2.3 (Elstrodt et al. [3, p. 105]). All the elements expressed in (2.1) form a representative system of all the $\Gamma$-conjugacy classes of hyperbolic elements of $\Gamma$.

Thanks to Lemmas 2.1-2.3, we can compute the contribution of semisimple conjugacy classes to the trace formula by the same method as that in [3]. The conclusions are in the following Lemmas 2.4 and 2.5.

Lemma 2.4. For an elliptic $\Gamma$-conjugacy class $R$ conjugate to $\left(\begin{array}{cc}\zeta & 0 \\ 0 & \zeta^{-1}\end{array}\right)$ in $G$ with $|\zeta|=1$, we have the following equality:

$$
\int_{\Gamma_{R} \backslash \mathbf{H}} k(v, R v) d v=\frac{\log N\left(T_{0}\right)}{\operatorname{ord} M(R)\left|1-\zeta^{2}\right|^{2}} g(0),
$$

where $M(R)$ is the maximal finite subgroup of $\Gamma_{R}$, and $T_{0}$ is a hyperbolic element defined in Lemma 2.1 .

Lemma 2.5. For a hyperbolic $\Gamma$-conjugacy class $T$ conjugate to $\left(\begin{array}{cc}a(T) & 0 \\ 0 & a(T)^{-1}\end{array}\right)$ in $G$ with $|a(T)|>1$, we have the following equality:

$$
\int_{\Gamma_{T} \backslash \mathbf{H}} k(v, R v) d v=\frac{\log N\left(T_{0}\right)}{\operatorname{ord}\left(\Gamma_{T}\right)_{\operatorname{tor}}\left|a(T)-a(T)^{-1}\right|^{2}} g(\log N(T)),
$$

where $T_{0}$ is defined in Lemma 2.2 .

The most mysterious part in the trace formula is the one concerning the continuous spectrum. Its contribution is known to be expressed in terms of Eisenstein series, which is defined as follows. For the time being we denote $\Gamma$ to be a cofinite discrete subgroup of $G$. Let $k_{1}:=\infty, k_{2}, \ldots, k_{h}$ be a complete set of inequivalent cusps, and let $\Gamma_{i}$ be the subgroup of $\Gamma$ that fixes $k_{i}$. Let $\rho_{i} \in G$ be the element such that $\rho_{i}\left(k_{i}\right)=\infty$ and that

$$
\rho_{i} \Gamma_{i} \rho_{i}^{-1}=\left\{\left(\begin{array}{ll}
1 & b \\
0 & 1
\end{array}\right) \mid b \in L_{i}\right\},
$$

where $L_{i}$ is a lattice in $\mathbf{C}$ such that $\operatorname{vol}\left(\mathbf{C} / L_{i}\right)=1$. For $v=z+y j \in \mathbf{H}$, we denote $v_{i}, z_{i}$, and $y_{i}$ by $\rho_{i} v=v_{i}=z_{i}+y_{i} j$. We denote $y_{i}$ as a mapping on $\mathbf{H}$ to $\mathbf{R}_{+}$for which $v \in \mathbf{H}$ corresponds to the coefficient of $j$ in $\rho_{i} v$. Then for each cusp $k_{i}$, define its Eisenstein series to be

$$
E_{i}(v, s):=\sum_{\gamma \in \Gamma_{i} \backslash \Gamma} y_{i}(\gamma v)^{s} \quad(\operatorname{Re}(s)>2) .
$$

It is known that $E_{i}(v, s)$ can be meromorphically continued in $s$ to the whole complex plane and that it admits a Fourier expansion at a cusp $k_{j}$, which is of the form

$$
E_{i}(v, s)=\delta_{i j} y_{j}^{s}+\varphi_{i j}(s) y_{j}^{2-s}+\cdots,
$$


where the remaining terms decay rapidly as $y_{j} \rightarrow \infty$. The matrix $\Phi(s):=$ $\left(\varphi_{i j}(s)\right)$ is called the scattering matrix with respect to $\Gamma$. For the Selberg trace formula, we need $\varphi(s):=\operatorname{det} \Phi(s)$, which we call the scattering determinant. Its form is not known in general. Here again we restrict $\Gamma$ as in $\S 1$. For the present $\Gamma$, Efrat and Sarnak smartly proved the following theorems without any explicit computation of constant terms of Eisenstein series.

Theorem 2.6 (Efrat and Sarnak [2, Theorem 1]). For $\Gamma=\operatorname{PSL}\left(2, O_{K}\right)$, the scattering determinant is

$$
\varphi(s)=(-1)^{\left(h-2^{t-1}\right) / 2} \omega_{K}^{2 s-2} \frac{\hat{\zeta}_{H}(s-1)}{\hat{\zeta}_{H}(s)},
$$

where $h$ is the class number of $K, \omega_{K}:=\sqrt{2} / d_{K}^{1 / 4}, d_{K}$ is the absolute value of the discriminant of $K, \hat{\zeta}_{H}(s):=\left(d_{H}^{1 / 2} /(2 \pi)^{h}\right)^{s} \Gamma(s)^{h} \zeta_{H}(s), H$ is the Hilbert class field of $K$, and $t$ is the number of prime divisors of $d_{K}$.

Theorem 2.7 [2, Theorem 2]. Let $t$ be as in the previous theorem. Then $\operatorname{tr}(\Phi(1))$ $=2^{t-1}-2$.

Now we can write down the Selberg trace formula.

Proposition 2.8. If we express the Selberg trace formula as

$$
\operatorname{Tr}_{D}+\operatorname{Tr}_{C}=I+E+H+P,
$$

each term has the following explicit form:

(2.5.H) $\quad H=\sum_{T \in \mathbf{P}} \sum_{n=1}^{\infty} \sum_{m=0}^{\nu_{T}-1} \frac{\log N(T)}{\nu_{T}} \frac{g(n \log (N(T)))}{\left|a(T)^{n} \zeta(T)^{m}-a(T)^{-n} \zeta(T)^{-m}\right|^{2}}$, 


$$
\begin{gathered}
P_{1}=\frac{h}{4 \pi}\left(\frac{C}{R}+3 \log 2-2 \gamma\right) g(0), \\
P_{2}=\frac{h}{4} h(1), \\
P_{3}=-\frac{h}{2 \pi} \int_{-\infty}^{\infty} h\left(r^{2}+1\right) \frac{\Gamma^{\prime}}{\Gamma}(1+i r) d r,
\end{gathered}
$$

where $\operatorname{Tr}_{C}=\sum_{i=1}^{4} \operatorname{Tr}_{C_{i}}$ and $P=\sum_{i=1}^{3} P_{i}$. The constants with respect to $K$ are as in Theorem 2.6. In (2.5.C3), a runs through all the integral ideals of the Hilbert class field of $K$, and $\Lambda(\mathbf{a})$ is the von Mangoldt function, equal to $\log N(\mathbf{p})$ if $\mathbf{a}$ is a power of a prime ideal $\mathbf{p}$, and zero otherwise. In (2.5.C4), $\kappa_{H}$ is the residue of $\zeta_{H}(s)$ at $s=1$. In (2.5.E) and $(2.5 . \mathrm{H}), R(\operatorname{resp} . T)$ runs through all the primitive elliptic (resp. hyperbolic) conjugacy classes of $\Gamma$, and $\nu_{R}\left(\right.$ resp. $\left.\nu_{T}\right)$ is the order of $R$ (resp. $R_{0}$ in (2.1)). The constants $C$ and $R$ are the constant term and the residue of some Epstein zeta function with respect to $\Gamma$ at $s=1$, whose explicit form is given in the proof below. The Euler constant is denoted by $\gamma$.

Proof. Semisimple terms $E$ and $H$ are obtained from Lemmas 2.4 and 2.5. It suffices to show the proof for the terms $\operatorname{Tr}_{C}, I$, and $P$. We will apply the general theory of Warner in [10], which is reviewed in [4]. First we treat $\operatorname{Tr}_{C}$. It turns out from [4, Theorem 1.2], that

$$
\operatorname{Tr}_{C}=-\frac{1}{4 \pi} \int_{-\infty}^{\infty} \frac{\varphi^{\prime}}{\varphi}(1+i r) h\left(r^{2}+1\right) d r+\frac{h(1)}{4} \operatorname{tr}(\Phi(1)) .
$$

As the scattering determinant $\varphi(s)$ is given in Theorem 2.6, we can compute the first term in (2.6) in the same way as in [5, p. 509]. The second term in (2.6) is equal to $-h(1)\left(2^{t-1}-2\right) / 4$ by Theorem 2.7 . Next, for the identity term $I$, we have the formula in $[4,(1.13),(1.14)$, Theorem 1.2], which says

$$
I=\frac{\operatorname{vol}(\Gamma \backslash \mathbf{H})}{4 \pi} \int_{-\infty}^{\infty} h\left(r^{2}+1\right) c(r)^{-1} c(-r)^{-1} d r,
$$

where

$$
c(r)^{-1}:=\frac{\Gamma((p+q) / 2) \Gamma(i r+p / 2) \Gamma(i r / 2+(p+2 q) / 4)}{\Gamma(p+q) \Gamma(i r) \Gamma(i r / 2+p / 4)},
$$

with nonnegative integers $p$ and $q$ decided in the process described in [4]. In this case an elementary computation of Lie algebra tells us that $p=2$ and $q=0$, which shows that $c(r)^{-1}=i r$ and gives (2.5.I). The parabolic terms are given by (2.5.P1)-(2.5.P3) in [10], because the number of cusps is now equal to $h$. The notations $C$ and $R$ are the constant term and the residue of the Epstein zeta function

$$
\zeta_{\Gamma}(s):=\sum_{X \in \log (\Gamma \cap N)-\{0\}} \frac{1}{\|X\|^{2 s}}
$$

at $s=1$, where $N$ is the nilpotent part of the Iwasawa decomposition of $G$,

$$
N=\left\{\left(\begin{array}{ll}
1 & b \\
0 & 0
\end{array}\right) ; b \in \mathbf{C}\right\}
$$


and $\|\cdot\|$ is the norm induced by the Killing form. We have

$$
\log (\Gamma \cap N)=\left\{\left(\begin{array}{ll}
0 & b \\
0 & 0
\end{array}\right) ; b \in O_{K}\right\}
$$

and if we put $X=\left(\begin{array}{ll}0 & b \\ 0 & 0\end{array}\right) \in \log (\Gamma \cap N)$, it is computed that $\|X\|^{2}=4|b|^{2}$. The zeta function turns out to be

$$
\zeta_{\Gamma}(s)=\sum_{b \in O_{K}^{\times}} \frac{1}{\left(4|b|^{2}\right)^{s}} .
$$

Now Chowla-Selberg's formula gives the explicit form of $C$ and $R$ :

$$
\begin{aligned}
C= & \frac{\pi^{2}}{12}+\frac{\varepsilon \pi \gamma}{2 \sqrt{D}}+\frac{\sqrt{\varepsilon} \pi}{D^{1 / 4}} \sum_{n=1}^{\infty} \sqrt{n} \sigma_{-1}(n)(-1)^{(\varepsilon-1) n} \\
& \times \int_{0}^{\infty} t^{-1 / 2} \exp \left(-\frac{\pi n \sqrt{D}}{\varepsilon}\left(t+t^{-1}\right)\right) d t
\end{aligned}
$$

and

$$
R=\frac{\varepsilon \pi}{4 \sqrt{D}}, \quad \text { where } \quad \varepsilon:=\left\{\begin{array}{ll}
1 & (D \not \equiv 3(\bmod 4)), \\
2 & (D \equiv 3(\bmod 4)),
\end{array} \quad \sigma_{-1}(n):=\sum_{d \mid n} d^{-1}\right.
$$

\section{THE DETERMINANT OF THE LAPLACIAN}

In this section we will define the determinant of the Laplacian composed of both discrete and continuous spectrum. First, we treat the discrete part $\operatorname{det}_{D}$. For this purpose, we introduce the spectral zeta function generalized by a variable $s$ :

$$
\zeta(w, s, \Delta):=\sum_{n=0}^{\infty} \frac{1}{\left(\lambda_{n}+s(s-2)\right)^{w}} .
$$

Elstrodt et al. [3, Corollary 1.5] show that it converges at least in $\operatorname{Re}(w)>2$ when $\Gamma$ is cocompact. Our method is similar to the case $G=\operatorname{PSL}(2, \mathbf{R})$ in [6].

Theorem 3.1. The spectral zeta function has the analytic continuation to the whole w-plane except the following poles:

$$
\begin{array}{lll}
w=\frac{3}{2}, & & \text { order } 1, \\
w=\frac{1}{2}-n \quad(n=0,1,2, \ldots), & & \text { order } 2 .
\end{array}
$$

In particular, it is holomorphic at $w=0$.

Proof. As the test function of the Selberg trace formula, we adopt the following function:

$$
h\left(r^{2}+1\right):=\exp \left(-t\left(r^{2}+1+s(s-2)\right)\right) \quad(t>0, s>2),
$$

which satisfies the assumptions of the trace formula. The corresponding $g(u)$ is

$$
g(u)=\frac{1}{\sqrt{4 \pi t}} \exp \left(-t(s-1)^{2}-\frac{u^{2}}{4 t}\right)
$$


Then the spectral zeta function $\zeta(w, s, \Delta)$ appears in the Mellin transformation of $\operatorname{Tr}_{D}$;

$$
\int_{0}^{\infty} \sum_{n=0}^{\infty} \exp \left(-t\left(\lambda_{n}+s(s-2)\right)\right) t^{w} \frac{d t}{t}=\Gamma(w) \zeta(w, s, \Delta) .
$$

So we give the analytic continuation to the Mellin transformation of each term of the trace formula. Poles of $\zeta(w, s, \Delta)$ are the points where the above integral diverges. As $t \rightarrow \infty$, there is no problem because $\operatorname{Tr}_{D}(t)$ decays exponentially. All we have to do is examine the behavior of each term as $t \rightarrow 0$. First, $I(t)$ is computed as follows:

$$
\begin{aligned}
I(t) & =\frac{\operatorname{vol}(\Gamma \backslash \mathbf{H})}{4 \pi} \int_{-\infty}^{\infty} \exp \left(-t\left(r^{2}+1+s(s-2)\right)\right) r^{2} d r \\
& =\frac{\operatorname{vol}(\Gamma \backslash \mathbf{H})}{4 \pi} \exp \left(-t(s-1)^{2}\right) t^{-3 / 2} \Gamma\left(\frac{3}{2}\right)=\sum_{n=0}^{\infty} a_{n} t^{n-3 / 2} .
\end{aligned}
$$

Next, $E(t), P_{1}(t)$, and $\operatorname{Tr}_{C 1}(t)$ are directly expanded as a power series of $t$ :

$$
E(t)+P_{1}(t)-\operatorname{Tr}_{C 1}(t)=\sum_{n=0}^{\infty} b_{n} t^{n-1 / 2}
$$

Similarly, $\operatorname{Tr}_{C 4}(t)$ and $P_{2}(t)$ are expanded as

$$
P_{2}(t)-\operatorname{Tr}_{C 4}(t)=\sum_{n=0}^{\infty} c_{n} t^{n}
$$

It is obvious that $H(t)$ and $\operatorname{Tr}_{C 3}(t)$ are exponentially small as $t \rightarrow 0$. Expansions obtained so far cause simple poles of $\zeta(w, s, \Delta)$ at $w=\frac{3}{2}-n$ $(n=0,1,2, \ldots)$, because

$$
\int_{0}^{1} t^{a} t^{w-1} d t=\frac{1}{w+a} \quad(\operatorname{Re} w>-a)
$$

for the behavior $t^{a} \quad(a \in \mathbf{R})$, and when $a$ is a nonnegative integer, it contributes the corresponding pole of $\Gamma(w)$. Now $P_{3}(t)$ and $\operatorname{Tr}_{C 2}(t)$ remain. We can apply the method in [6], which is originally due to Kurokawa [8]. We will directly compute the poles of $M\left(P_{3}(t)-\operatorname{Tr}_{C 2}(t)\right)(w)$, where $M$ denotes the Mellin transformation. It is equal to

$$
\begin{aligned}
-\frac{h}{\pi} & \int_{-\infty}^{\infty} \int_{0}^{\infty} \exp \left(-t\left(r^{2}+(s-1)^{2}\right)\right) t^{w} \frac{d t}{t} \psi(1+i r) d r \\
= & -\frac{h}{\pi} \Gamma(w) \int_{-\infty}^{\infty}\left(r^{2}+(s-1)^{2}\right)^{-w} \psi(1+i r) d r \\
= & -\frac{2 h}{\pi} \Gamma(w)\left(\int_{1}^{\infty}\left(r^{2}+(s-1)^{2}\right)^{-w} \operatorname{Re}(\psi(1+i r)) d r+(\text { entire })\right) \\
=-\frac{h}{\pi} \Gamma(w)\left((s-1)^{1-2 w} \int_{0}^{1}(1+y)^{-w} y^{w-3 / 2}\right. & \left.\quad \times \operatorname{Re}\left(\psi\left(1+\frac{(s-1) i}{\sqrt{y}}\right)\right) d y+(\text { entire })\right) \quad\left(r=\frac{s-1}{\sqrt{y}}\right) .
\end{aligned}
$$


The Stirling-Binet formula $[11,12.3$, p. 252] shows that

$$
\operatorname{Re}(\psi(1+i r))=\log r+\sum_{n=1}^{N} \frac{\alpha_{n}}{r^{2 n}}+R_{N}(r) \quad\left(\alpha_{n} \in \mathbf{R}\right),
$$

with $\left|R_{N}(r)\right| \leq M_{n} / r^{2 N+1} \quad\left(r \geq \frac{1}{2}\right)$, where the $\alpha_{n}$ are constants expressed via Bernoulli numbers. Moreover, we can apply the binomial expansion formula to get $(y+1)^{-w}=\sum_{k=0}^{\infty}\left(\begin{array}{c}-w \\ k\end{array}\right) y^{k}$. Then $M\left(P_{3}(t)-\operatorname{Tr}_{C 2}(t)\right)(w)$ is equal to

$$
\begin{aligned}
&-\frac{h}{\pi} \Gamma(w)(s-1)^{1-2 w}\left(\sum_{k=0}^{\infty}\left(\begin{array}{c}
-w \\
k
\end{array}\right) \int_{0}^{1} y^{k+w-3 / 2}\right. \\
&\left.\times\left(-\frac{\log y}{2}+\sum_{n=1}^{N} \alpha_{n} y^{n}+R_{N}\left(y^{-1 / 2}\right)\right) d y+(\text { entire })\right) \\
&=-\frac{h}{\pi} \Gamma(w)(s-1)^{1-2 w}\left(\sum _ { k = 0 } ^ { \infty } ( \begin{array} { c } 
{ - w } \\
{ k }
\end{array} ) \left(-\frac{1}{2}\left(k+w-\frac{1}{2}\right)^{-2}\right.\right. \\
&\left.\left.+\sum_{n=1}^{\infty} \alpha_{n}\left(n+k+w-\frac{1}{2}\right)^{-1}\right)+(\text { entire })\right) .
\end{aligned}
$$

Therefore $M\left(P_{3}(t)-\operatorname{Tr}_{C 2}(t)\right)(w)$ is expressed as

$$
M\left(P_{3}(t)-\operatorname{Tr}_{C 2}(t)\right)(w)=\Gamma(w)\left(\sum_{k=0}^{\infty}\left(\frac{p_{k}(w)}{\left(w+k-\frac{1}{2}\right)^{2}}+\frac{q_{k}(w)}{w+k-\frac{1}{2}}\right)+r(w)\right),
$$

where $p_{k}(w), q_{k}(w)(k=0,1,2, \ldots$,$) and r(w)$ are holomorphic functions on the whole $w$-plane. These terms cause double poles of $\zeta(w, s, \Delta)$ at $w=$ $\frac{1}{2}-n(n=0,1,2, \ldots)$. The proof is complete.

Now we can define the discrete part of the determinant of the Laplacian.

\section{Definition 3.2.}

$$
\operatorname{det}_{D}(\Delta+s(s-2)):=\exp \left(-\left.\frac{\partial}{\partial w}\right|_{w=0} \zeta(w, s, \Delta)\right) .
$$

Formally, it is $\prod_{n=0}^{\infty}\left(\lambda_{n}+s(s-2)\right)$.

In the proof of Theorem 3.1 we expanded all terms in the trace formula as $t \rightarrow 0$ except for $P_{3}(t)-\operatorname{Tr}_{C 2}(t)$. Its expansion is obtained by the inverse Mellin transformation.

Proposition 3.3. As $t \rightarrow 0$,

$$
P_{3}(t)-\operatorname{Tr}_{C 2}(t)=\sum_{n=0}^{\infty} d_{n} t^{n}+\sum_{n=0}^{\infty}\left(e_{n}+f_{n} \log t\right) t^{n-1 / 2} .
$$

Proof. The inverse Mellin transformation shows that

$$
P_{3}(t)-\operatorname{Tr}_{C 2}(t)=\frac{1}{2 \pi i} \int_{c-i \infty}^{c+i \infty} M\left(P_{3}(t)-\operatorname{Tr}_{C 2}(t)\right)(w) t^{-w} d w \quad\left(\operatorname{Re}(c)>\frac{1}{2}\right)
$$


The integrand has double poles at $w=\frac{1}{2}-n \quad(n=0,1,2, \ldots)$, and simple poles at $w=-n(n=0,1,2, \ldots)$. The residue at $w=\frac{1}{2}-n$ is $\lim _{w \rightarrow 1 / 2-n} \frac{d}{d w}\left(\left(w+n+\frac{1}{2}\right)^{2} M\left(P_{3}(t)-\operatorname{Tr}_{C 2}(t)\right)(w) t^{-w}\right)=\left(e_{n}+f_{n} \log t\right)^{n-1 / 2}$, where

$$
e_{n}=\Gamma^{\prime}\left(\frac{1}{2}-n\right) p_{n}\left(\frac{1}{2}-n\right)+\Gamma\left(\frac{1}{2}-n\right)\left(p_{n}^{\prime}\left(\frac{1}{2}-n\right)+q_{n}\left(\frac{1}{2}-n\right)\right)
$$

and

$$
f_{n}=-\Gamma\left(\frac{1}{2}-n\right) p_{n}\left(\frac{1}{2}-n\right) .
$$

Simple poles come from $\Gamma(w)$. The residue at $w=-n(n=0,1,2, \ldots)$ is

$$
\lim _{w \rightarrow-n}\left((w+n) M C P_{3}(w) t^{-w}\right)=d_{n} t^{n},
$$

where

$$
d_{n}=\frac{1}{(-1)^{n} n !} \sum_{k=0}^{\infty}\left(\frac{p_{k}(-n)}{\left(-n+k-\frac{1}{2}\right)^{2}}+\frac{q_{k}(-n)}{-n+k-\frac{1}{2}}\right)+r(-n) .
$$

Comparing the integral with the integral along the rectangle,

$$
c-i T \rightarrow c+i T \rightarrow-(N+\varepsilon)+i T \rightarrow-(N+\varepsilon)-i T \rightarrow c-i T
$$

$$
(T, \varepsilon, N>0, N \in \mathbf{Z}) \text {, }
$$

and letting $N \rightarrow \infty$, we obtain the desired expansion.

Here we take a new test function for the Selberg trace formula:

$$
h\left(r^{2}+1\right):=\frac{1}{r^{2}+1+s(s-2)}-\frac{1}{r^{2}+\beta^{2}} \quad\left(\beta>\frac{1}{2}, s>2\right),
$$

and let

$$
g(u)=\frac{1}{2 s-2} e^{-(s-1)|u|}-\frac{1}{2 \beta} e^{-\beta|u|} .
$$

All terms in the trace formula can be regarded as functions of $s$. Then it turns out the following relation is valid:

$$
\frac{d}{d s} \operatorname{Tr}_{D}(s)=\frac{d}{d s} \frac{1}{2 s-2} \frac{d}{d s} \log \operatorname{det}_{D}(\Delta+s(s-2)) .
$$

So we define the contribution of the continuous spectrum to the determinant of the Laplacian as

$$
\frac{d}{d s} \operatorname{Tr}_{C i}(s)=\frac{d}{d s} \frac{1}{2 s-2} \frac{d}{d s} \log \operatorname{det}_{C i}(\Delta, s) \quad(i=1,2,3,4) .
$$

By solving the above differential equation, we get the continuous part of the determinant of the Laplacian.

Proposition 3.4. The contribution of the continuous spectrum to the determinant of the Laplacian is the product of the following.

$$
\begin{aligned}
& \operatorname{det}_{C 1}(\Delta, s)=\left(\omega_{K}(2 \pi)^{h} / d_{H}^{1 / 2}\right)^{-s}, \\
& \operatorname{det}_{C 2}(\Delta, s)=\Gamma(s)^{h}, \\
& \operatorname{det}_{C 3}(\Delta, s)=\zeta_{H}(s), \\
& \operatorname{det}_{C 4}(\Delta, s)=(s-1)^{\kappa_{H}+\left(2^{t-1}-2\right) / 2} .
\end{aligned}
$$


Proof. We obtain (3.1.1) and (3.1.4) easily by an elementary calculation. It is also easy to get (3.1.3) considering that the logarithmic derivative of the Dedekind zeta function is $-\sum_{\mathrm{a}} \Lambda(\mathbf{a}) / N(\mathbf{a})$, where the condition on a and the function $\Lambda$ is as in Proposition 2.8. As for (3.1.2), we compute the integral in $\operatorname{Tr}_{C 2}(s)$ by comparing the one along the lower half-circle and the real axis. It has only one pole at $r=-(s-1) i$ in the region, which is simple. The residue is $i \frac{\Gamma^{\prime}}{\Gamma}(s)$. Then (3.1.2) is obvious and the proof is finished.

\section{The local Selberg zeta functions}

Here again we take the test function

$$
h\left(r^{2}+1\right):=\frac{1}{r^{2}+1+s(s-2)}-\frac{1}{r^{2}+\beta^{2}} \quad\left(\beta>\frac{1}{2}, s>2\right),
$$

and let

$$
g(u)=\frac{1}{2 s-2} e^{-(s-1)|u|}-\frac{1}{2 \beta} e^{-\beta|u|} .
$$

Definition 4.1. The Selberg zeta function for the present $G$ and $\Gamma$ is defined as

$$
Z(s):=\prod_{T \in \mathbf{P}} \prod_{k \equiv l\left(\bmod \nu_{T}\right)}\left(1-a(T)^{-2 k} \overline{a(T)}^{-2 l} N(T)^{-s}\right),
$$

where $k$ and $l$ run through all the nonnegative integers satisfying the congruence relation.

Lemma 4.2. The Selberg zeta function (4.1) satisfies

$$
\frac{d}{d s} H(s)=\frac{d}{d s} \frac{1}{2 s-2} \frac{d}{d s} \log Z(s),
$$

where $H(s)$ is the hyperbolic term of the trace formula regarded as a function of $s$, and $a(T)$ is the eigenvalue of $T$ such that $|a(T)|>1$ and $N(T):=|a(T)|^{2}$. Proof. We need to carry out a standard calculation which is similar, for example, to that in [3].

We will express the derivation of each term of the trace formula as

$$
\frac{d}{d s} \frac{1}{2 s-2} \frac{d}{d s} \log X(s),
$$

with some function $X(s)$, which will be called local Selberg zeta functions. For $I(s), E(s)$, and $P_{i}(s)$, the corresponding local zeta functions will be denoted by $Z_{I}(s), Z_{E}(s)$, and $Z_{P_{i}}(s) \quad(i=1,2,3)$.

Proposition 4.3. The local Selberg zeta functions for $G$ and $\Gamma$ are the following:

$$
Z_{I}(s)=\exp \left(-\frac{\operatorname{vol}(\Gamma \backslash \mathbf{H})}{6}(s-1)^{3}\right)
$$

$$
Z_{E}(s)=\exp \left(\sum_{R} \frac{\log N\left(T_{0}\right)}{2 \operatorname{ord} M(R)} \sum_{m=0}^{\nu_{R}-1}\left(1-\cos \frac{2 m \pi}{\nu_{R}}\right)^{-1} s\right),
$$

(4.2.P2) $Z_{P_{2}}(s)=(s-1)^{h / 2}$,

(4.2.P3) $\quad Z_{P_{3}}(s)=\Gamma(s)^{-h}$. 
Proof. It is enough to carry out an elementary calculation on all the functions except for $Z_{P_{3}}$. For $Z_{P_{3}}$, we only need to compute by calculating residues in a lower half-circle.

From Definition 3.2 and Propositions 3.4 and 4.3, we can deduce the main result:

Theorem 4.4. For the present $G$ and $\Gamma$, the Selberg zeta function $Z(s)$ has the following determinant expression for some constants $c$ and $c^{\prime}$ :

$$
\widehat{Z}(s)=e^{c-c^{\prime} s(2-s)} \operatorname{det}(\Delta, s),
$$

where $\hat{Z}(s):=Z_{I}(s) Z_{E}(s) Z(s) Z_{P}(s)$ and

$$
\operatorname{det}(\Delta, s):=\operatorname{det}_{D}(\Delta+s(s-2)) \operatorname{det}_{C}(\Delta, s),
$$

whose factors are given explicitly by Proposition 4.3, Definition 3.2, and Proposition 3.4.

\section{Decision of The CONSTANTS}

In this section, we determine the constants $c$ and $c^{\prime}$ in (4.3). Taking the logarithm of (4.3), we have

$$
\begin{aligned}
& \log Z_{I}(s)+\log Z_{E}(s)+\log Z(s)+\log Z_{P}(s) \\
& \quad=c+c^{\prime} s(s-2)+\log \operatorname{det}_{D}(\Delta+s(s-2))+\log \operatorname{det}_{C}(\Delta, s) .
\end{aligned}
$$

We will compare the behavior of both sides of $(5.1)$ as $s \rightarrow \infty$. From Proposition 4.3 and using Stirling's formula for $\log Z_{P_{3}}(s)$, we have

$$
\begin{aligned}
\log Z_{I}(s) & =-\frac{\operatorname{vol}(\Gamma \backslash \mathbf{H})}{6}(s-1)^{3}, \\
\log Z_{E}(s) & =\sum_{R} \frac{\log N\left(T_{0}\right)}{2 \operatorname{ord} M(R)} \sum_{m=0}^{\nu_{R}-1}\left(1-\cos \frac{2 m \pi}{\nu_{R}}\right)^{-1} s, \\
\log Z_{P_{1}}(s) & =\frac{h}{4 \pi}\left(\frac{C}{R}+3 \log 2-2 \gamma\right) s, \\
\log Z_{P_{2}}(s) & =\frac{h}{2} \log (s-1), \\
\log Z_{P_{3}}(s) & =-h\left(\frac{\log 2 \pi}{2}+\left(s-\frac{1}{2}\right) \log s-s+o(1)\right) .
\end{aligned}
$$

Similarly, from Proposition 3.3 we have

(5.2.C1) $\log \operatorname{det}_{C 1}(\Delta, s)=-s \log \frac{\omega_{K}(2 \pi)^{h}}{d_{H}^{1 / 2}}$,

(5.2.C2) $\log \operatorname{det}_{C 2}(\Delta, s)=h\left(\frac{\log 2 \pi}{2}+\left(s-\frac{1}{2}\right) \log s-s+o(1)\right)$,

(5.2.C4) $\log \operatorname{det}_{C 4}(\Delta, s)=\kappa_{H}+\frac{2^{t-1}-2}{2} \log (s-1)$,

and $\log \operatorname{det}_{C_{3}}(\Delta, s)$ is exponentially small as $s \rightarrow \infty$. It remains to decide the behavior of $\log \operatorname{det}_{D}(\Delta+s(s-2))$. We apply the method of Sarnak [9]. We put 
$\operatorname{Tr}_{0}(t):=\sum_{n=0}^{\infty} e^{-t \lambda_{n}}$, which is $\operatorname{Tr}_{D}(t)$ with $s \rightarrow 2$. By the proof of Theorem 3.1 ,

$$
\operatorname{Tr}_{0}(t)=\sum_{n=0}^{\infty} A_{n} t^{n}+\sum_{n=0}^{\infty} B_{n} t^{n-3 / 2}+\sum_{n=0}^{\infty} C_{n} t^{n-1 / 2} \log t
$$

for some constants $A_{n}, B_{n}$, and $C_{n}$. Let

$$
f(t):=\left(\operatorname{Tr}_{0}(t)-A_{0}-\sum_{n=0}^{2} B_{n} t^{n-3 / 2}-\sum_{n=0}^{1} C_{n} t^{n-1 / 2} \log t\right) \times \frac{1}{t},
$$

which is bounded near $t=0$. We have

$$
\begin{aligned}
& \log \operatorname{det}_{D}(\Delta+s(s-2))=-\left.\frac{d}{d w}\right|_{w=0} \zeta(w, s, \Delta) \\
& =-\left.\frac{d}{d w}\right|_{w=0} \frac{1}{\Gamma(w)} \int_{0}^{1} \operatorname{Tr}_{0}(t) e^{-t s(s-2)} t^{w} \frac{d t}{t}+o(1) .
\end{aligned}
$$

We decompose $\operatorname{Tr}_{0}(t) \times \frac{1}{t}$ into $f(t)$ and other terms.

Lemma 5.1. For $a \in \mathbf{R}-\{0\}$, we have

$$
-\left.\frac{d}{d w}\right|_{w=0} \frac{1}{\Gamma(w)} \int_{0}^{1} t^{a+w} e^{-t s(s-2)} \frac{d t}{t}=-\frac{\Gamma(a)}{(s(s-2))^{a}}+o(1)
$$

as $s \rightarrow \infty$.

Proof.

$$
\begin{aligned}
-\frac{d}{d w} & \left.\right|_{w=0} \frac{1}{\Gamma(w)} \int_{0}^{1} t^{a} e^{-t s(s-2)} t^{w} \frac{d t}{t} \\
& =-\left.\frac{d}{d w}\right|_{w=0} \frac{1}{\Gamma(w)(s(s-2))^{a+w}} \int_{0}^{s(s-2)} t^{a+w} e^{-t} \frac{d t}{t} \\
& =-\left.\frac{d}{d w}\right|_{w=0} \frac{1}{\Gamma(w)(s(s-2))^{a+w}}\left(\Gamma(a+w)-\int_{s(s-2)}^{\infty} t^{a+w} e^{-t} \frac{d t}{t}\right) \\
& =-\frac{\Gamma(a)}{(s(s-2))^{a}}+o(1) . \quad \square
\end{aligned}
$$

Similarly, other terms in the expansion are treated as follows.

Lemma 5.2. As $s \rightarrow \infty$,

$$
-\left.\frac{d}{d w}\right|_{w=0} \frac{1}{\Gamma(w)} \int_{0}^{1} t^{w} e^{-t s(s-2)} \frac{d t}{t}=\log (s(s-2))+o(1) .
$$

Lemma 5.3. For $a \in \mathbf{R}-\{0\}$, we have

$$
\begin{aligned}
& -\left.\frac{d}{d w}\right|_{w=0} \frac{1}{\Gamma(w)} \int_{0}^{1} t^{a+w} e^{-t s(s-2)} \log t \frac{d t}{t} \\
& =-\frac{\Gamma^{\prime}(a)}{(s(s-2))^{a}}+\frac{\Gamma(a) \log (s(s-2))}{(s(s-2))^{a}}+o(1)
\end{aligned}
$$

as $s \rightarrow \infty$.

By the above three lemmas, we have the behavior of $\log \operatorname{det}_{D}(\Delta+s(s-2))$ as $s \rightarrow \infty$. 
Lemma 5.4. As $s \rightarrow \infty$,

$$
\begin{aligned}
\log \operatorname{det}_{D}(\Delta+s(s-2))= & A_{0} \log (s(s-2))-B_{0} \Gamma\left(-\frac{3}{2}\right)(s(s-2))^{3 / 2} \\
& -\left(B_{1} \Gamma\left(-\frac{1}{2}\right)+C_{0} \Gamma^{\prime}\left(-\frac{1}{2}\right)\right)(s(s-2))^{1 / 2} \\
& +C_{0} \Gamma\left(\frac{1}{2}\right)(s(s-2))^{1 / 2} \log (s(s-2))+o(1),
\end{aligned}
$$

where $A_{0}, B_{0}, B_{1}$, and $C_{0}$ are constants defined in (5.3).

Proof.

$$
\begin{aligned}
& \log \operatorname{det}_{D}(\Delta+s(s-2)) \\
& =-\left.\frac{d}{d w}\right|_{w=0} \frac{1}{\Gamma(w)} \int_{0}^{1} \operatorname{Tr}_{0}(t) e^{-t s(s-2)} t^{w} \frac{d t}{t}+o(1) \\
& \quad-\left.\frac{d}{d w}\right|_{w=0} \frac{1}{\Gamma(w)} \int_{0}^{1}\left(A_{0}+\sum_{n=0}^{2} B_{n} t^{n-3 / 2}\right. \\
& \left.\quad+\sum_{n=0}^{1} C_{n} t^{n-1 / 2} \log t\right) \frac{1}{t} e^{-t s(s-2)} t^{w} d t+o(1) \\
& =A_{0} \log (s(s-2))-\sum_{n=0}^{1} B_{n} \Gamma\left(n-\frac{3}{2}\right)(s(s-2))^{3 / 2-n} \\
& +C_{0}\left(-\Gamma^{\prime}\left(-\frac{1}{2}\right)(s(s-2))^{1 / 2}+\Gamma\left(\frac{1}{2}\right)(s(s-2))^{1 / 2} \log (s(s-2))\right)+o(1) .
\end{aligned}
$$

We need to determine the constants $A_{0}, B_{0}, B_{1}$, and $C_{0}$.

Lemma 5.5. The above constants are given by the following expressions:

$$
\begin{aligned}
A_{0}= & \frac{h}{4}-\frac{\kappa_{H}}{2}+\frac{2^{t-1}-2}{4}-\frac{h}{\pi}\left(4+\sum_{n=1}^{\infty} \frac{\alpha_{n}}{n-\frac{1}{2}}\right), \\
B_{0}= & \frac{\operatorname{vol}(\Gamma \backslash \mathbf{H})}{4 \pi} \Gamma\left(\frac{3}{2}\right), \\
B_{1}= & -\frac{\operatorname{vol}(\Gamma \backslash \mathbf{H})}{4 \pi} \Gamma\left(\frac{3}{2}\right)-\frac{h}{2 \pi} \Gamma^{\prime}\left(\frac{1}{2}\right) \\
& +\frac{1}{\sqrt{4 \pi}}\left(\sum_{R} \frac{\log N\left(T_{0}\right)}{2 \operatorname{ord} M(R)} \sum_{m=0}^{\nu_{R}-1}\left(1-\cos \frac{2 \pi m}{\nu_{R}}\right)^{-1}\right. \\
& \left.+\frac{h}{4 \pi}\left(\frac{C}{R}+3 \log 2-2 \gamma\right)+\log \frac{\omega_{K}(2 \pi)^{h}}{d_{H}^{1 / 2}}\right), \\
C_{0}= & \frac{h}{2 \pi} \Gamma\left(\frac{1}{2}\right) .
\end{aligned}
$$

Proof. First, $A_{0}$ is equal to $c_{0}+d_{0}$ with $s \rightarrow 2$ as in $\S 3$. As $c_{0}$ is the constant term of

$$
\begin{aligned}
P_{2}(t)-\operatorname{Tr}_{C 4}(t) & =\left(\frac{h}{4}-\frac{\kappa_{H}}{2}+\frac{2^{t-1}-2}{4}\right) h(1) \\
& =\left(\frac{h}{4}-\frac{\kappa_{H}}{2}+\frac{2^{t-1}-2}{4}\right) e^{-t}
\end{aligned}
$$


it follows that

$$
c_{0}=\frac{h}{4}-\frac{\kappa_{H}}{2}+\frac{2^{t-1}-2}{4} .
$$

Here $d_{0}$ is the constant term of

$$
P_{3}(t)-\operatorname{Tr}_{C 2}(t)=-\frac{h}{\pi} \int_{-\infty}^{\infty} h\left(r^{2}+1\right) \frac{\Gamma^{\prime}}{\Gamma}(1+i r) d r
$$

From the proof of Proposition 3.3, $d_{0}$ with $s \rightarrow 2$ is equal to

$$
\begin{aligned}
& \sum_{k=0}^{\infty}\left(\frac{p_{k}(0)}{\left(k-\frac{1}{2}\right)^{2}}+\frac{q_{k}(0)}{k-\frac{1}{2}}\right)+r(0)=\lim _{w \rightarrow 0} w M\left(P_{3}(t)-\operatorname{Tr}_{C 2}(t)\right)(w) \\
& \quad=-\frac{h}{\pi} \int_{0}^{1} y^{-3 / 2}\left(-\frac{\log y}{2}+\sum_{n=1}^{\infty} \alpha_{n} y^{n}\right) d y=-\frac{h}{\pi}\left(4+\sum_{n=1}^{\infty} \frac{\alpha_{n}}{n-\frac{1}{2}}\right) .
\end{aligned}
$$

Next, $B_{0}$ is equal to $a_{0}$ with $s \rightarrow 2$, which is $\frac{\operatorname{vol}(\Gamma \backslash \mathbf{H})}{4 \pi} \Gamma\left(\frac{3}{2}\right)$. The constant $B_{1}$ is equal to the sum $a_{1}+b_{0}+e_{0}$, among which

$$
\begin{gathered}
a_{1}=-\frac{\operatorname{vol}(\Gamma \backslash \mathbf{H})}{4 \pi} \Gamma\left(\frac{3}{2}\right) \\
b_{0}=\frac{1}{\sqrt{4 \pi}}\left(\sum_{R} \frac{\log N\left(T_{0}\right)}{2 \operatorname{ord} M(R)} \sum_{m=0}^{\nu_{R}-1}\left(1-\cos \frac{2 \pi m}{\nu_{R}}\right)^{-1}\right. \\
\left.+\frac{h}{4 \pi}\left(\frac{C}{R}+3 \log 2-2 \gamma\right)+\log \frac{\omega_{K}(2 \pi)^{h}}{d_{H}^{1 / 2}}\right),
\end{gathered}
$$

for it is the coefficient of $t^{-1 / 2}$ in

$$
\begin{aligned}
E(t)+ & P_{1}(t)-\operatorname{Tr}_{C 1}(t) \\
= & \left(\sum_{R} \frac{\log N\left(T_{0}\right)}{2 \operatorname{ord} M(R)} \sum_{m=0}^{\nu_{R}-1}\left(1-\cos \frac{2 \pi m}{\nu_{R}}\right)^{-1}\right. \\
& \left.\quad+\frac{h}{4 \pi}\left(\frac{C}{R}+3 \log 2-2 \gamma\right)+\log \frac{\omega_{K}(2 \pi)^{h}}{d_{H}^{1 / 2}}\right) g(0) \\
= & \left(\sum_{R} \frac{\log N\left(T_{0}\right)}{2 \operatorname{ord} M(R)} \sum_{m=0}^{\nu_{R}-1}\left(1-\cos \frac{2 \pi m}{\nu_{R}}\right)^{-1}\right. \\
& \left.\quad+\frac{h}{4 \pi}\left(\frac{C}{R}+3 \log 2-2 \gamma\right)+\log \frac{\omega_{K}(2 \pi)^{h}}{d_{H}^{1 / 2}}\right) \frac{e^{-t}}{\sqrt{4 \pi t}},
\end{aligned}
$$

and $e_{0}$ is given its explicit form in the proof of Proposition 3.3:

$$
e_{0}=\Gamma^{\prime}\left(\frac{1}{2}\right) p_{0}\left(\frac{1}{2}\right)+\Gamma\left(\frac{1}{2}\right)\left(p_{0}^{\prime}\left(\frac{1}{2}\right)+q_{0}\left(\frac{1}{2}\right)\right) .
$$

Now, as $p_{0}(w)$ with $s \rightarrow 2$ is the constant function $-h / 2 \pi$ and $q_{0}(w)=0$,

$$
e_{0}=-\frac{h}{2 \pi} \Gamma^{\prime}\left(\frac{1}{2}\right) \text {. }
$$


Last, we will compute $C_{0}$. It is equal to $f_{0}$ with $s \rightarrow 2$ as in 3 :

$$
C_{0}=-\Gamma\left(\frac{1}{2}\right) p_{0}\left(\frac{1}{2}\right)=\frac{h}{2 \pi} \Gamma\left(\frac{1}{2}\right) .
$$

Now we can express each term of (5.1) as a function of $s$ using the following lemma.

Lemma 5.6. Let $a$ be any real number. As $s \rightarrow \infty$,

$$
\begin{gathered}
\log (s+a)=\log s+o(1), \\
s \log (s+a)=s \log s+a+o(1), \\
\log (s(s-2))=2 \log s+o(1), \\
(s(s-2))^{3 / 2}=s^{3}-3 s^{2}+\frac{3}{2} s-\frac{1}{2}+o(1),
\end{gathered}
$$

and

$$
(s(s-2))^{1 / 2}=s-1+o(1) .
$$

Proof. All that is necessary is to carry out elementary calculations.

Proposition 5.7. The constants $c$ and $c^{\prime}$ in the determinant expression (4.3) are as follows:

$$
\begin{aligned}
c= & \sum_{R} \frac{\log N\left(T_{0}\right)}{2 \operatorname{ord} M(R)} \sum_{m=0}^{\nu_{R}-1}\left(1-\cos \frac{2 m \pi}{\nu_{R}}\right)^{-1}+\frac{h}{4 \pi}\left(\frac{C}{R}+3 \log 2-2 \gamma\right) \\
& +\log \frac{\omega_{K}}{d_{H}^{1 / 2}}-\kappa_{H}+2 \pi i \mathrm{Z}
\end{aligned}
$$

and $c^{\prime}=0$.

Proof. The expansion of both sides in (5.1) are given by (5.2.I)-(5.2.C4) and Lemma 5.4. Computing with the previous lemma, we can express them by $s$. Comparing the coefficient of $s^{2}$ and the constant term, we obtain the conclusion.

\section{THE COMPARISON WITH EFRAT'S DETERMINANT}

In [1], Efrat introduces the determinant of the Laplacian composed of both discrete and continuous spectrum in the case of $G=\operatorname{SL}(2, \mathbf{R})$ and $\Gamma$ its cofinite torsion-free subgroup. In this section, we reconstruct the results in the previous sections in the framework of Efrat. (Results of Part I [6] are seen from this point of view in Part III [7].) If we extend his definition of the determinant to the present case, it is as follows. Let $\Gamma$ be a cofinite subgroup of $\operatorname{PSL}(2, \mathrm{C})$. First we list three types of sequences concerning the spectrum of $\Delta$.

(1) The set $S_{1}$ of $s_{n} \in \mathbf{C}$ such that $s_{n}\left(2-s_{n}\right)=\lambda_{n}$, where $\lambda_{n}$ is the discrete spectrum of $\Delta$.

(2) The set $S_{2}$ of poles $\rho_{m}=\beta_{m}+i \gamma_{m}$ of $\varphi(s)$ with $\beta_{m}<2$.

(3) The set $S_{3}=\left\{\eta_{1}, \ldots, \eta_{N}\right\}$ of exceptional poles of $\varphi(s)$ in $(1,2]$.

The spectral zeta function $\zeta(w, s)$ is defined by

$$
\zeta(w, s):=\sum_{\sigma \in S}(\sigma(1-\sigma)-s(1-s))^{-w}
$$


where $S=S_{1} \cup S_{2}-S_{3}$. The determinant of the Laplacian is defined to be

$$
\operatorname{det}(\Delta-s(2-s))^{2}:=\exp \left(-\left.\frac{\partial}{\partial w}\right|_{w=0} \zeta(w, s)\right),
$$

after proving the regularity of $\zeta(w, s)$ at $w=0$. This definition is different from that in the previous sections. Indeed, the determinant expression of the Selberg zeta function is

$$
\operatorname{det}(\Delta-s(2-s))^{2}=\varphi(s) \hat{Z}(s)^{2} \times(\text { gamma } \sim \text { factor }) .
$$

Now let $\Gamma$ be as in the previous sections. Theorem 2.6 leads us to the explicit form of the sets $S_{2}$ and $S_{3}$. The set $S_{2}$ is the sequence of all the nontrivial zeros of $\hat{\zeta}_{H}(s)$ and $S_{3}=\{2\}$. We define the spectral zeta function for each sequence by

$$
\zeta_{i}(w, s):=\sum_{\sigma \in S_{i}}(\sigma(1-\sigma)-s(1-s))^{-w} \quad(i=1,2,3) .
$$

We have the regularity of $\zeta_{1}(w, s)$ at $w=0$ by the same proof as that of Theorem 3.1, which shows that the discrete and the continuous part of the determinant exist separately in the framework of Efrat. The continuous part of the determinant of the Laplacian is defined by

$$
\operatorname{det}_{C}(\Delta-s(2-s)):=\frac{\operatorname{det}_{2}(\Delta-s(2-s))}{\operatorname{det}_{3}(\Delta-s(2-s))},
$$

where

$$
\operatorname{det}_{i}(\Delta-s(2-s)):=\exp \left(-\left.\frac{\partial}{\partial w}\right|_{w=0} \zeta_{i}(w, s)\right) .
$$

Theorem 6.1. For the present $\Gamma$, the continuous part of the determinant of the Laplacian is expressed by

$$
\operatorname{det}_{C}(\Delta-s(1-s))^{2}=e^{d-d^{\prime} s(2-s)} \hat{\zeta}_{H}(s) \hat{\zeta}_{H}(s-1)(s-1)^{2}
$$

with some constants $d$ and $d^{\prime}$.

Proof. The function $s(s-1) \hat{\zeta}_{H}(s)$ is entire and has the following expression as an infinite product:

$$
s(s-1) \hat{\zeta}_{H}(s)=p e^{\alpha s} \prod_{\rho \in S_{2}}\left(1-\frac{s}{\rho}\right) e^{s / \rho},
$$

with some constants $p$ and $\alpha$. We have the identity

$$
\begin{aligned}
& \frac{d}{d s} \frac{1}{2 s-2} \frac{d}{d s} \log \operatorname{det}_{2}(\Delta-s(2-s))^{2} \\
& \quad=\frac{d}{d s} \frac{1}{2 s-2} \frac{d}{d s} \log \hat{\zeta}_{H}(s) \hat{\zeta}_{H}(s-1)(s-1)^{2} s(s-2) .
\end{aligned}
$$

Indeed, a little calculation shows that both sides are equal to

$$
-\sum_{\rho \in S_{2}} \frac{2 s-2}{((\rho-s)(\rho-(2-s)))^{2}} .
$$

On the other hand, it is easy to compute that

$$
\operatorname{det}_{3}(\Delta-s(2-s))^{2}=s(s-2) \text {. }
$$

The proof is complete. 
The constants $d$ and $d^{\prime}$ in Theorem 6.1 can be computed by making explicit the theorem of Efrat in this case.

\section{REFERENCES}

1. I. Efrat, Determinant of Laplacians on surfaces of finite volume, Comm. Math. Phys. 119 (1988), 443-451.

2. I. Efrat and P. Sarnak, The determinant of the Eisenstein matrix and Hilbert class fields, Trans. Amer. Math. Soc. 290 (1985), 815-824.

3. J. Elstrodt, F. Grunewald, and J. Mennicke, The Selberg zeta function for cocompact discrete subgroups of PSL $(2$, C), Banach Center Publ., no. 17, PWN, 1985, pp. 83-120.

4. R. Gangolli and G. Warner, Zeta functions of Selberg's type for some non-compact quotients of symmetric spaces of rank one, Nagoya Math. J. 78 (1980), 1-44.

5. D. A. Hejhal, The Selberg trace formula for $\operatorname{PSL}(2, \mathbf{R})$, Vol. 2, Lecture Notes in Math., vol. 1001, Springer, 1983.

6. S. Koyama, Determinant expression of Selberg zeta functions. I, Trans. Amer. Math. Soc. 324 (1991), 149-168.

7. _ Determinant expression of Selberg zeta functions. III, Proc. Amer. Math. Soc. 113 (1991), 303-311.

8. N. Kurokawa, Parabolic components of zeta functions, Proc. Japan Acad. Ser. A Math. Sci. 64 (1988), 21-24.

9. P. Sarnak, Determinants of Laplacians, Comm. Math. Phys. 110 (1987), 113-120.

10. G. Warner, Selberg's trace formula for nonuniform lattices: The R-rank one case, Adv. Math. Suppl. Stud. 6 (1979), 1-142.

11. E. T. Whittaker and G. N. Watson, A course of modern analysis, Cambridge Univ. Press, Cambridge, 1927.

Department of Mathematics, Tokyo Institute of Technology, Oh-Okayama, Meguro$\mathrm{Ku}$, TOKYO, 152, JAPAN

Current address: Department of Mathematics, Faculty of Science and Technology, Keio University, 14-1, Hiyoshi, 3 chome, Kohoku-ku, Yokohama 223, Japan 